\title{
Spreadsheet assisted overall Design of a Wind Turbine Blade
}

\author{
U. Aguirre Llona ${ }^{1}$, J. J. Pérez Rambla ${ }^{2}$ and G. Aguirre Zamalloa ${ }^{1}$ \\ ${ }^{1}$ Department of Electrical Engineering \\ E.S.I.B., Bilbao, University of Basque Country \\ Alda Urquijo s/n, 48013 Bilbao (Spain) \\ Phone:+34 946014057, Fax:+34 946014200, e-mail: iepagzag@lg.ehu.es \\ ${ }^{2}$ Ebro-Cantábrica de Energías Renovables (ECERSA) \\ c/Albert Einstein 15, Ed. CEIA, Of. 126 \\ Parque Tecnológico de Álava, 01510 Miñano, Álava (Spain) \\ Phone: +34 945 298207, enorsa@enorsa.pt-alava.es
}

\begin{abstract}
In this paper we show in an orderly, step by step fashion the typical hypotheses, approximations and tradeoffs that go into the design of a wind turbine blade. The main objective of the work is not an efficient code but a clear one. We discuss the interplay between different strategies and we illustrate the procedure with a real blade calculation. Both aerodynamic and mechanic design are addressed.
\end{abstract}

\section{Key words}

BEM, Blade design, Excel, HAWT, NACA, pitch control, WT_perf.

\section{Introduction}

The very Art of the design engineer belongs to the proper account of the relevant factors at work in some machine or device, to the balancing of the physical and mathematical approximations, and to their harmonious integration within a sensible model, and all of this with due regard to economic feasibility.

It is quite fair to state that Renewable Energy Technologies are one of the most demanding disciplines on account of the broad range of competences they call for from the design engineer; an excellent example is provided by the intertwined aerodynamic, mechanic and electric problems to be solved by the design of a wind turbine.

In this work we present a step by step bottom-up design methodology based on the Blade Element Theory (BEM) for the profiling of the blades of a horizontal upwind wind turbine. Anyhow, we do not claim it to be the "best" possible design procedure; we think that is a reasonably simple design procedure and that any designer should give it a try as a first approximation of a "real" design. The motivation behind this work is twofold:

a) We wanted to organise the calculations within a clear, ready to be coded pattern. b) We wanted to give an illustrative and interesting detailed example thereof.

At this point we want to stress the difference between design and simulation. Design, as we understand it, pertains to the proper profiling and sizing of the different elements composing the assembly under scrutiny, and its natural setting is the (time independent) steady state analysis. On the other hand, finite element method based simulation, as implemented by industrial strength codes, solves the true time dependent (transient) rigorous equations of the system. Here we are not dealing with vibrations, fatigue calculations, gyroscopic forces nor yawing moments on the structure. However, our analysis must precede such advanced calculations.

Because of the great volume of data we will be dealing with, calculations ought to be done with the help of a computer, be it with a spreadsheet or with a scripted programming language. We think that it is pointless to give here the whole source code of the program. Rather, we prefer to describe with some detail its organisation and purpose.

As mentioned, analysis means problem decomposition, which in turn means that every analysis is ad hoc to some extent. The procedure advocated here has nevertheless the advantage of being fully iterative; this special layout lends itself to improvement because the hypotheses introduced at each step are clearly and purposely stated.

At any rate, it is worth stressing that an aerodynamic design is a complex, highly non-linear task which calls for the use of a computer and great finesse on the part of the analyst. So much so that, in practice, that the indicated steps or "iterations" interact an get inextricably mixed. This is unavoidable and makes the beauty of the craft. Sometimes the analyst may be guided by some empirical rules, such as "the pressure centre lies at $1 / 3$ of the radius" or "the centre of pressure as near as possible from the gravity centre". These "principles" can only be implemented on a try and error basis. 
The paper is organised as follows: the first section is this Introduction. Then in the second section we state the Design Goals, the independent set of variables of our choice, and we start the iterative selection of the airfoil type and radius. Third section is devoted to the aerodynamic calculations. In the subsection A we present the theoretical background, and in the next B subsection we perform the iterative calculation of an "optimised" chord. Subsection C deals with the complete blade calculation at rated wind speed. Then in the subsections $\mathrm{D}$ and $\mathrm{E}$ we tackle the power, torque and thrust calculations at speeds lower and higher than rated wind speed, respectively. To end with this section we present some special cases (wind gusts and feathered position) in the F subsection. Next section (4) has to do with the mechanical design of the steel beam (which is our innermost iterative loop). The main conclusions come in section 5 and next a handful of References is included.

\section{Design Goals}

First of all we establish our design goals: we want to design a two bladed, blade-pitch controlled, variable velocity and constant tip-speed ratio wind generator of $\mathrm{P}=200 \mathrm{~kW}$ rated power. The machines developed by ECERSA are provided with an oil hydraulic system of power transmission which make them very rugged and subject to low maintenance costs. On the other hand the typical overall efficiency reached is about $\eta \approx 0.87$. We will calculate the power output, torque and thrust at wind speeds $5<\mathrm{V}<20 \mathrm{~m} / \mathrm{s}$ with rated speed $\mathrm{V}_{\mathrm{r}}=12 \mathrm{~m} / \mathrm{s}$ (we disregard the effect of wind shear). We will analyse an incompressible flow with (sea level air density) $\rho=\rho_{\text {air }}=1.25 \mathrm{~kg} / \mathrm{m}^{3}$. In correspondence with the blade number $b=2$ choice, we fix the tip speed to wind speed ratio as $\lambda_{\text {design }}=6$. [1]-[3].

A decision as to the airfoil choice has to be done at this stage. Several high performance profiles are in common use: NACA series 4412, 4415, 4418 and Göttingen 398 for instance, to name just a few. In a fully iterative procedure this is naturally the start of the first iteration loop. Several profiles should be utilised and the final results compared (power-torque-weigh-cost) to make an informed decision. For illustrative purposes it will suffice to show here the results concerning the choice of NACA 4415 airfoil, which is well known to offer good performances [3].

Now, we estimate an average power coefficient $\mathrm{C}_{\mathrm{p}} \approx 0.45$. Here starts the second iterative loop: the radius of the disk swept by the machine depends on this choice and has to be calculated in a self consistent way because the $\mathrm{C}_{\mathrm{p}}$ coefficient is recalculated at a later stage:

$$
R=r_{\text {max }}=\sqrt{\frac{2 \cdot P}{\eta \cdot \rho \cdot \pi \cdot \mathrm{V}_{r}^{3} \cdot C_{p}}}=12.27 \mathrm{~m}
$$

also

$$
\omega=\frac{\lambda_{\text {design }} \cdot \mathrm{V}_{r}}{\mathrm{r}_{\max }}=5.87 \frac{\mathrm{rad}}{\mathrm{s}}
$$

As it stands and will be discussed below, there is still one last degree of freedom that should be fixed by the designer: the incidence angle.

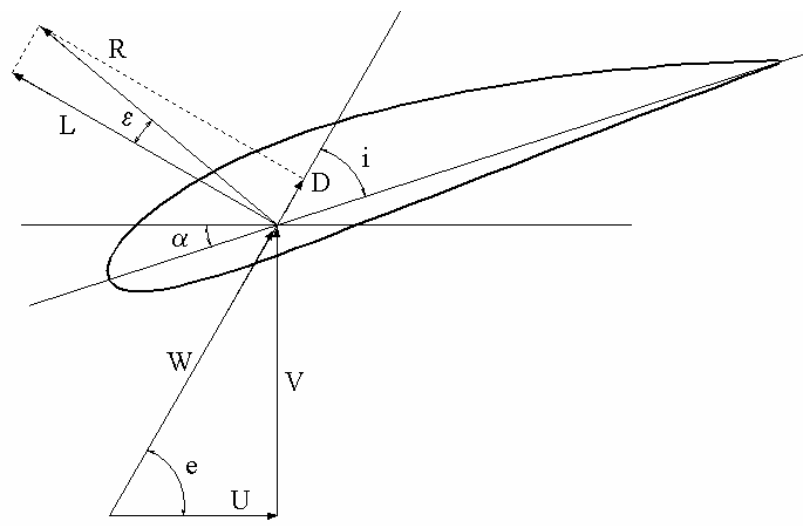

Fig. 1: Airfoil setting and notation

\section{Aerodynamic Calculations}

First of all a word about notation: in the BEM method a discretisation is performed, but we feel free to indicate all magnitudes interchangeably with continuous or discrete indexes, with differentials or with increments. We will write for instance $f(r)$ or $f_{i}=f\left(r_{i}\right)$ where $r_{i}=r_{\min }+i \cdot \Delta r$, with $r_{\text {min }}=r_{H u b}, \Delta r$ the discretisation step and $0 \leq i \leq N=\frac{r_{\max }-r_{\min }}{\Delta r}$. Also, the notation utilised throughout for speeds, forces and angles should be clarified (see Fig.1):

a) Wind velocity vectors:

$\mathrm{U}$ : apparent wind induced by the tangential velocity $\left(\left.\vec{U}\right|_{\text {wind }}=-\left.\vec{U}\right|_{\text {blade motion }}\right)$. If we disregard induction effects it is given by $U=\omega \cdot r$. V: absolute wind velocity (we admit here that it is an homogeneous field). $\mathrm{W}$ : relative wind velocity for an observer moving with the blade. Vectorially $\vec{W}=\vec{V}+\left(-\vec{U}_{\text {motion }}\right)=\vec{V}+\vec{U}_{\text {wind }}$.

b) Forces:

$\mathrm{L}=\mathrm{F}_{\mathrm{L}}\left(\mathrm{dL}=\mathrm{dF}_{\mathrm{L}}\right.$ for differential elements $)$ stand for Lift. $\mathrm{D}=\mathrm{F}_{\mathrm{D}}\left(\mathrm{dD}=\mathrm{dF}_{\mathrm{D}}\right.$ for differential elements $)$ stand for Drag. $\mathrm{R}=\mathrm{F}_{\mathrm{R}} \quad\left(\mathrm{dR}=\mathrm{dF}_{\mathrm{R}}\right.$ for differential elements $)$ stand for resultant (total) force.

\section{c) Angles:}

$i(r)$ is the incidence angle; $\alpha(r)$ is the setting angle (it comprises twist and pitch); $e(r)=\tan ^{-1}\left(\frac{V}{U}\right)$ is the inclination angle. Inspection of Figure 1 makes clear that:

$$
e=\alpha+i
$$

Moreover 


$$
\varepsilon(r)=\tan ^{-1}\left(\frac{D}{L}\right)=\tan ^{-1}\left(\frac{C_{D}}{C_{L}}\right)
$$

where $C_{L}(i)$ and $C_{D}(i)$ are the lift and drag coefficients of the NACA 4415 airfoil, taken from [3]

\section{A. Theoretical background}

The vortex theory of Glauert [3], [5] defines two coefficients $\mathrm{h}(\mathrm{r})$ and $\mathrm{k}(\mathrm{r})$ which take into account the induced rotation of the airflow which goes through the rotor provoked by the sheet of vortices originated at the tip and the hub of the finite blade. In this theory the axial velocity becomes:

$$
V=\frac{1+k}{2} \cdot V_{1}
$$

where $\mathrm{V}_{1}$ corresponds to the upstream, unperturbed wind velocity. Clearly in the ideal Betz limit we have $k=\frac{1}{3}$. And the tangential velocity becomes:

$$
U=\frac{1+h}{2} \cdot \omega \cdot r
$$

If the vortex induction is negligible we have: $h=1$. On the other hand:

$$
W=\sqrt{U^{2}+V^{2}}
$$

Now, we can calculate the elementary axial force (thrust) either by the aerodynamic expression:

$$
d F_{a}=\frac{1}{2} \cdot \rho \cdot b \cdot c(r) \cdot W^{2} \cdot C_{L} \cdot \frac{\cos (e-\varepsilon)}{\cos \varepsilon} \cdot d r
$$

where $c(r)$ is the blade chord, or by application of axial momentum conservation:

$$
d F_{a}=\rho \cdot \pi \cdot r \cdot V_{1}^{2} \cdot\left(1-k^{2}\right) \cdot d r
$$

In parallel, we can calculate the elementary tangential force (torque producing: $d T=r \cdot d F_{t}$ ) either by the aerodynamic expression:

$$
d F_{t}=\frac{1}{2} \cdot \rho \cdot b \cdot c(r) \cdot W^{2} \cdot C_{L} \cdot \frac{\sin (e-\varepsilon)}{\cos \varepsilon} \cdot d r
$$

or by application of angular momentum conservation:

$$
d F_{t}=\rho \cdot \pi \cdot r^{2} \cdot \omega \cdot V_{1} \cdot(1+k) \cdot(h-1) \cdot d r
$$

The expression of the local power coefficient is:

$$
C_{p}=\frac{\omega \cdot r \cdot \frac{d F_{t}}{d r}}{\pi \cdot r \cdot \rho \cdot V_{1}^{3}}=\lambda^{2} \cdot(k+1) \cdot(h-1)
$$

where the shorthand $\lambda(r)=\frac{r \cdot \omega}{V_{1}}$ has been used. We have now enough elements to perform the actual calculations

\section{B. Ideal and real chords}

In this section we explain how to obtain an "optimised" chord, which amounts to perform a third iteration. Examining the equations above we realise that the fundamental $i(r), \alpha(r)$ (or $e(r))$ and $c(r)$ quantities are unknown. Also, we realise that only two of them are independent. We need an extra bit of information to obtain the chord. Hence, we set up the following model: it is not hard to demonstrate [3] that an aerodynamically "smart" choice of $i(r)$ is that which makes $\tan (\varepsilon)$ a minimum because it maximises the local $C_{p}$ (aerodynamic efficiency) at the element. Actually this angle is $i_{o} \approx 6^{\circ}$ for the NACA4415 aerofoil. The ideal minimum is $\tan (\varepsilon)=0$. With this ideal assumption and equating and combining the above equations we get the following expressions:

$$
h(k)=\sqrt{1+\frac{1-k^{2}}{\lambda^{2}}}
$$

and

$$
\tan (e)=\frac{1}{\lambda} \cdot \frac{1+k}{1+h}
$$

Now, we choose k such that $C_{p}(k)$ is a local maximum:

$$
\frac{d C_{p}}{d k}=0 \Leftrightarrow k=\sqrt{1+\lambda^{2}} \cdot \cos \left(\frac{\pi+\tan ^{-1}(\lambda)}{3}\right)
$$

The ideal chord can now be calculated, for instance from:

$$
c(r)=\frac{8 \cdot \pi \cdot r \cdot(1-k) \cdot \sin ^{2}(e)}{b \cdot C_{L}(i) \cdot(1+k) \cdot \cos (e)}
$$

This ideal chord is illustrated in Figure 2.

Two remarks are in order, however. First, the manufacture of a blade with such a curve may not be possible or economically interesting. This is why we have adopted a piecewise linear approximation which closely resembles the ideal chord (see Fig.2). Second, setting $i=i_{o}$ is certainly not the mechanically best solution. This value of $i$ leads to unacceptably large chords near the hub (increased weigh) A compromise must be sought, increasing $i$ near the hub such that the aerodynamical performance is not drastically diminished but the chord shrinks to reasonable values. This is the reason of our choice of $i(r)$ shown in Figure 3. 


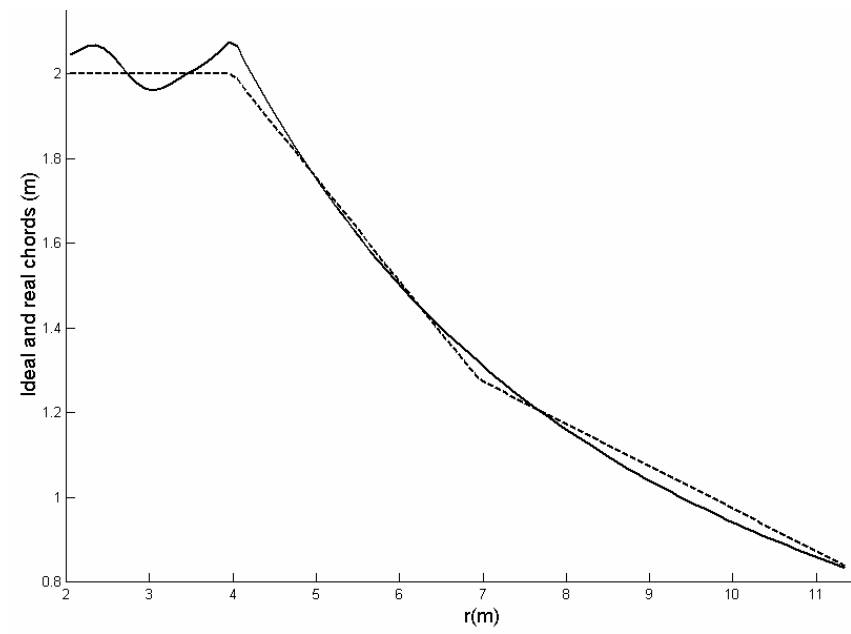

Figure 2: Ideal and PWL approximate real chords

\section{Rated wind speed calculations}

Once $c(r)$ and $i(r)$ are known and fixed, we drop the ideal assumption $\tan (\varepsilon)=0$ and come back to equations (5)-(11). We have to solve simultaneously this set of nonlinear equations at rated wind speed (fourth iterative loop) to calculate: $d F_{a}(r), d F_{t}(r), e(r), h(r)$ and $k(r)$. At each element we iterate starting from the guesses $k=\frac{1}{3}$ and $h=1$; with these values we use (8) and (10) to calculate the forces and again (9) and (11) to recalculate the induction coefficients, until convergence is achieved.

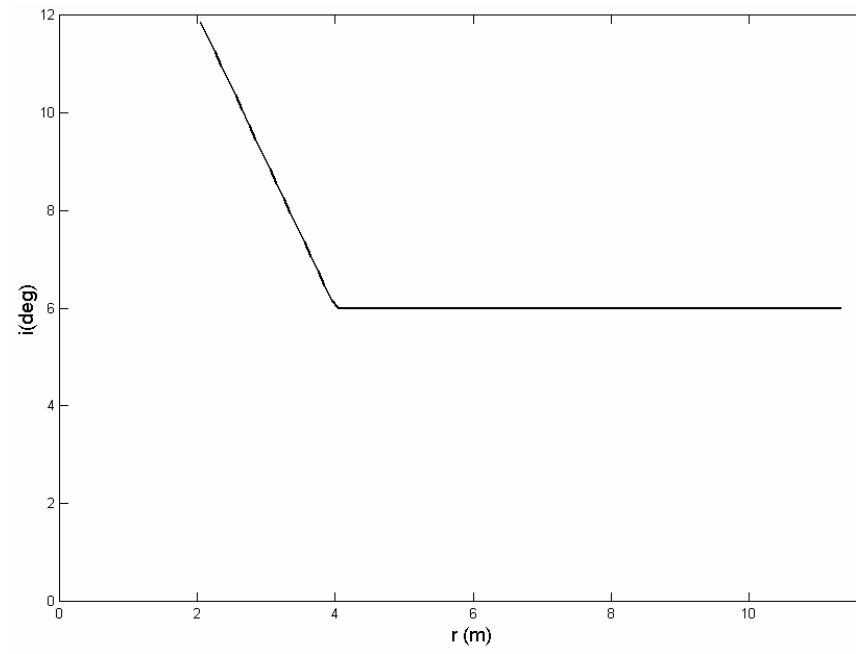

Figure 3: design incidence angle at rated speed

Apart from the values of power, thrust and torque illustrated in Figure 6, the most interesting result of this calculation is the twist distribution of the blade given by the calculated $\left.\alpha(r)\right|_{V-\text { rated }}$ that is illustrated in Figure 4 along with a smooth cubic interpolant ("real" $\alpha$ ) that we will keep for good in calculations at speeds other than rated.

\section{Calculations at wind speeds lower than rated}

This is the fifth iterative loop. We know the chord $c, \alpha$ that we keep constant and just calculate the forces $d F_{a}(r), d F_{t}(r)$, the induction coefficients $h(r)$ and $k(r)$, and the new incidence $i(r)$ (or $e(r))$ for different

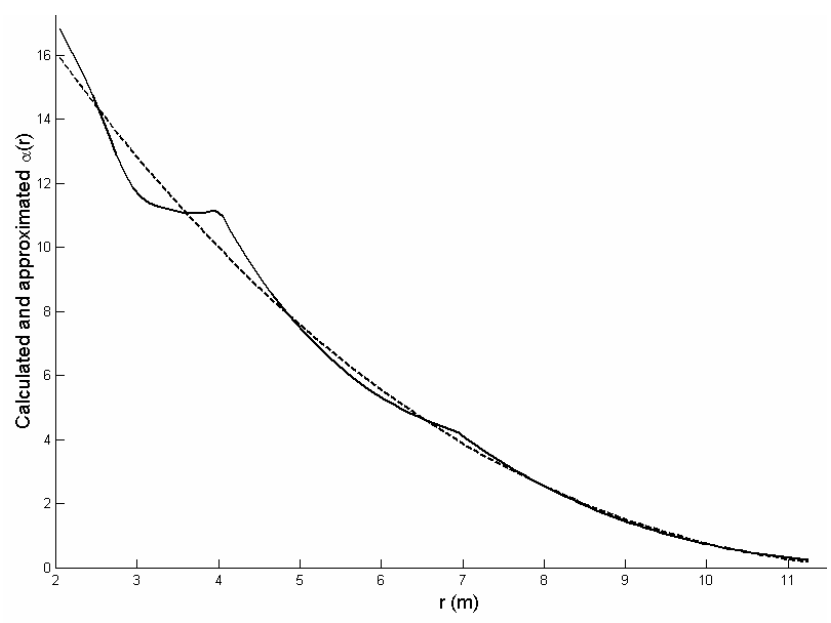

Figure 4: calculated and approximated $\alpha(r)$

wind speeds below rated. Results are summarised in Figure 6.

\section{E. Calculations at wind speeds higher than rated}

This is the sixth iterative loop. The principle of calculation is very much the same as in the precedent subsection. The only difference is that a pitch angle is introduced to keep the power at rated values:

$$
\alpha(r)=\alpha_{\text {real }}(r)+\Delta \alpha
$$

These values are illustrated in Figure 5.

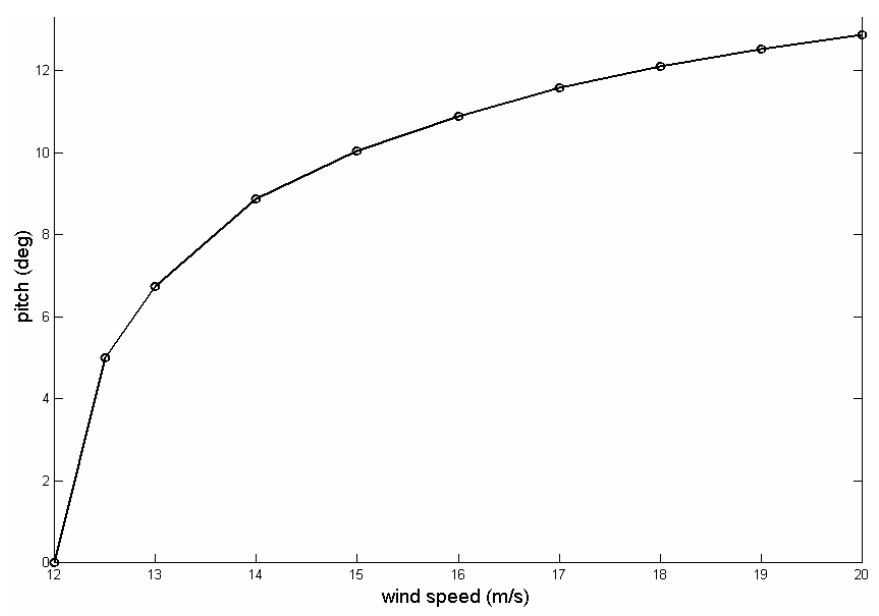

Figure 5: Variation of pitch angle (deg) for steady rated power

\section{F. Some special cases}

Once we have the code up and running, it is in principle possible to simulate any situation and calculate thrust, power and torque even in abnormal situations; especially interesting are, for example, the instantaneous wind 
gusts, say, of $\mathrm{V}=30 \mathrm{~m} / \mathrm{s}$ or higher at rated omega with pitch angle zero or higher, for example $80^{\circ}$, near the feather position. However these calculations are only meaningful as far as $C_{L}$ and $C_{D}$ coefficients measured (in a wind tunnel) over a wide enough $i$ range become available. Otherwise we can make some approximations: we can naively pose $C_{L}=0$ and $C_{D}=1$ each time that the incidence angle is out of bounds. A better solution (implemented for example in the WT_perf code) is to use data from flat plate theory.
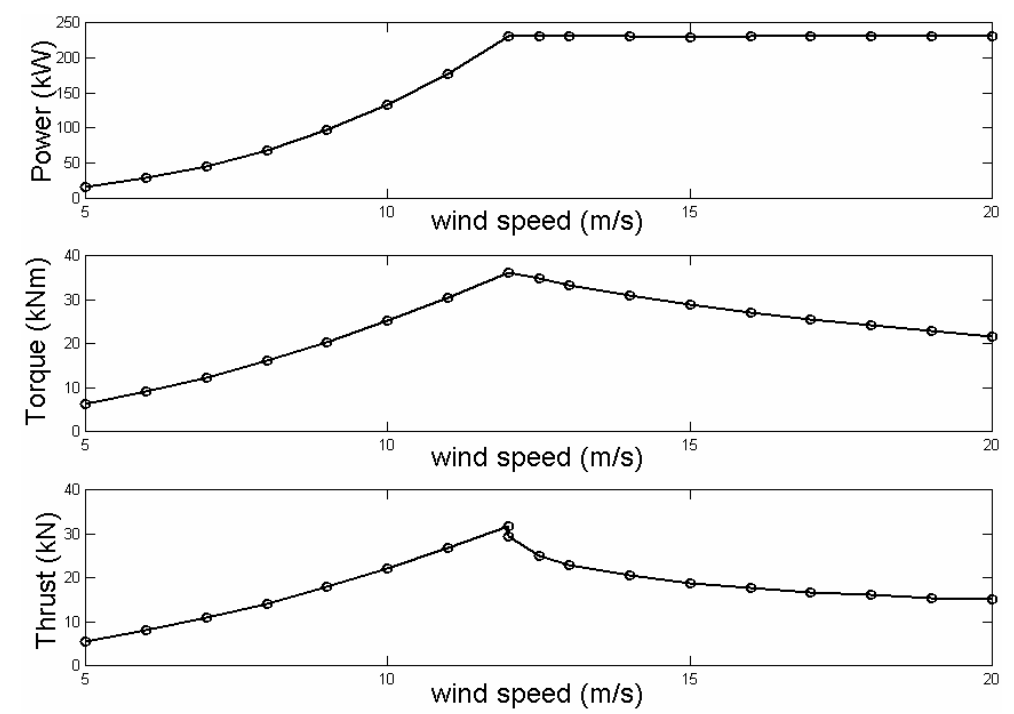

Figure 6: Results of the aerodynamic calculations

\section{Mechanic Calculations}

This is the innermost iterative loop our calculation. Our goal is to calculate the diameters of the cylindrically hollow steel beam that will be able to stand its own weigh in addition to the aerodynamic efforts calculated beforehand. The variable that we have to optimise is the weigh of the beam. Ours being a static calculation we will concentrate only in one position: that with the blades horizontal, so that the bending moment due to their weigh is worst. We arbitrarily divide the total length in $\mathrm{N}$ pieces, say 4 or 5 of equal length and start calculating the proper sizes of these pieces from the tip down to the hub. If the procedure does not produce a useful result we will have to revise this partitioning (consider more pieces and/or of variable length). The properties of the steel in use are: $\rho_{\text {steel }}=7850 \mathrm{~kg} / \mathrm{m}^{3}$ and $\sigma_{\max }=510 \mathrm{MPa}$ (maximum stress). The maximum external diameter (D) of each piece is given by $\mathrm{D}_{\max }=0.15^{*}$ chord at the highest radius in a NACA 4415 aerofoil. The inner diameter (d) can be calculated with the help of the following expression:

$$
f \cdot \sigma_{\max }=\frac{M}{I} \cdot r_{\max }
$$

where $I=\frac{\pi}{64} \cdot\left(D^{4}-d^{4}\right)$ is the surface moment of a hollow cylinder, $r_{\max }=\frac{D}{2}, f$ is a security factor, say $f=0.65$ for instance, and $\mathrm{M}$ is the total bending moment at the section. Here we are assuming that the elemental force total lies in the same plane, independent of $r$. This approximate value of $M$ is in fact an overestimation of the real value so that we are on the safe side. In order to obtain $\mathrm{M}$ we apply:

$$
M(r)=\int_{r}^{r \max }(x-r) \cdot \frac{d R_{T}}{d x}(x) \cdot d x
$$

where

$$
d R_{T}=\sqrt{\left(d R_{A} \cdot \sin (e-\varepsilon)+d P\right)^{2}+\left(d R_{A} \cdot \cos (e-\varepsilon)\right)^{2}}
$$

$d R_{A}$ is the aerodynamic resultant at the element, $d R_{T}$ is the grand total and $d P$ is the weigh of the element. The weigh of the fibreglass and of the other materials is altogether negligible in comparison with the steel beam. The total bending moment calculated with (19) is shown in Figure 7. The results obtained with this method after some tinkering are illustrated in Table 1.

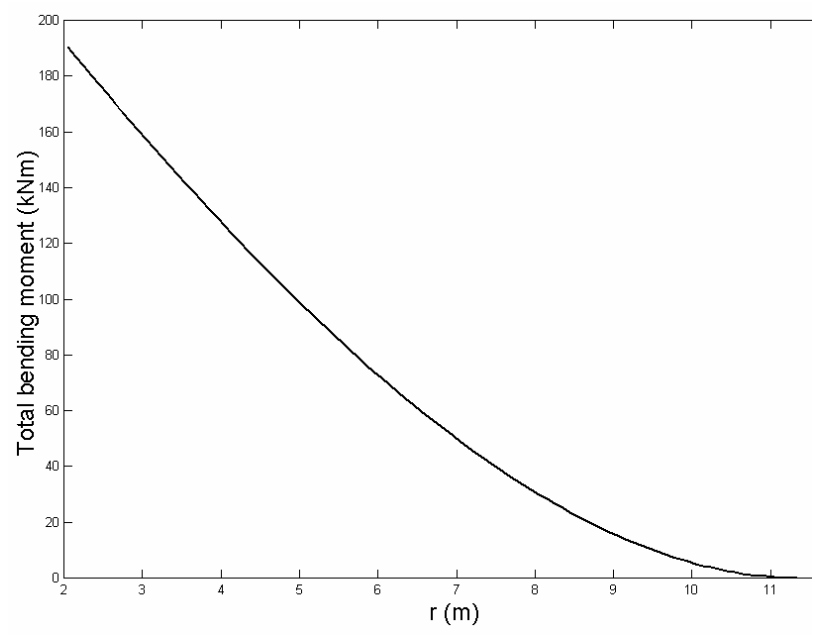

Figure 7: Total bending moment over the beam

Table 1: Sizing of the steel beam (see text)

\begin{tabular}{|l|l|l|l|l|l|l|}
\hline & $\mathrm{D}_{\max }$ & $\mathrm{D}(\mathrm{cm})$ & $\begin{array}{l}\mathrm{d} \\
(\mathrm{cm})\end{array}$ & $\begin{array}{l}\text { Mass } \\
(\mathrm{kg})\end{array}$ & $\sigma(\mathrm{MPa})$ & $f \cdot \sigma_{\max }$ \\
\hline piece 1 & 28.7 & 28.4 & 26.3 & 162.9 & 319.9 & 332 \\
\hline piece 2 & 20.3 & 20 & 17.2 & 147.7 & 329.8 & 332 \\
\hline piece 3 & 16.2 & 16 & 13.9 & 89.0 & 332.9 & 332 \\
\hline piece 4 & 12.6 & 12 & 11 & 34.0 & 329.7 & 332 \\
\hline Total & & & & 433.7 & & \\
\hline
\end{tabular}

\section{Conclusions}

We present a quite generic blade design procedure implemented with a spreadsheet, which is highly scalable and can suit the design of blades of differing sizes. This methodology is capable of yielding a good preliminary blade design by taking into account both aerodynamic and dynamic factors influencing the blade performance.

\section{References}

[1] D. Le Gouriérès, Wind Power Plants; Theory and Design, Pergamon Press, Oxford (1982), pp. 76-120. 
[2] T. Burton, D. Sharpe, N. Jenkins and E. Bossanyi, Handbook of Wind Energy, John Wiley \& Sons, LTD, Chichester (2001).

[3] E. Hau, Wind Turbines, Springer Verlag, Berlin (2000), pp. 69-211
[4] Marshall L. Buhl Jr., WT_perf User's Guide, National Wind Technology Center, NREL, Golden, Colorado (2000). http://wind.nrel.gov/designcodes/wtperf/

[5] Glauert H. Airplane propellers. Vol 4 DNL in Aerodynamic Theory. Edited by Durand W. F., Dover ed. (1943) 